\title{
Weighted Differentiation Composition Operators to Bloch-Type Spaces
}

\author{
Junming Liu, ${ }^{1}$ Zengjian Lou, ${ }^{1}$ and Ajay K. Sharma ${ }^{2}$ \\ ${ }^{1}$ Department of Mathematics, Shantou University, Shantou, Guangdong 515063, China \\ ${ }^{2}$ School of Mathematics, Shri Mata Vaishno Devi University, Kakryal, Katra 182320, India \\ Correspondence should be addressed to Zengjian Lou; zjlou@stu.edu.cn
}

Received 2 February 2013; Accepted 8 April 2013

Academic Editor: Pedro M. Lima

Copyright (C) 2013 Junming Liu et al. This is an open access article distributed under the Creative Commons Attribution License, which permits unrestricted use, distribution, and reproduction in any medium, provided the original work is properly cited.

\begin{abstract}
We characterized the boundedness and compactness of weighted differentiation composition operators from BMOA and the Bloch space to Bloch-type spaces. Moreover, we obtain new characterizations of boundedness and compactness of weighted differentiation composition operators.
\end{abstract}

\section{Introduction}

Let $\mathbb{D}$ be the open unit disk in the complex plane $\mathbb{C}, H(\mathbb{D})$ the space of all functions holomorphic on $\mathbb{D}, d A(z)=(1 / \pi) d x d y$ the normalized area measure on $\mathbb{D}$, and $H^{\infty}$ the space of all bounded holomorphic functions with the norm $\|f\|_{\infty}=$ $\sup _{z \in \mathbb{D}}|f(z)|$.

Let $\alpha>0$. The $\alpha$-Bloch space $\mathscr{B}^{\alpha}$ on $\mathbb{D}$ is the space of all holomorphic functions $f$ on $\mathbb{D}$ such that

$$
\sup _{z \in \mathbb{D}}\left(1-|z|^{2}\right)^{\alpha}\left|f^{\prime}(z)\right|<\infty .
$$

The little $\alpha$-Bloch space $\mathscr{B}_{0}^{\alpha}$ consists of all $f \in \mathscr{B}^{\alpha}$ such that

$$
\lim _{|z| \rightarrow 1}\left(1-|z|^{2}\right)^{\alpha}\left|f^{\prime}(z)\right|=0 .
$$

Both spaces $\mathscr{B}^{\alpha}$ and $\mathscr{B}_{0}^{\alpha}$ are Banach spaces with the norm

$$
\|f\|_{\mathscr{B}^{\alpha}}=|f(0)|+\sup _{z \in \mathbb{D}}\left(1-|z|^{2}\right)^{\alpha}\left|f^{\prime}(z)\right|,
$$

and $\mathscr{B}_{0}^{\alpha}$ is a closed subspace of $\mathscr{B}^{\alpha}$. If $\alpha=1$, they become the classical Bloch space $\mathscr{B}$ and little Bloch space $\mathscr{B}_{0}$, respectively. For any $\alpha>0$, the space $\mathscr{A}_{\infty}^{\alpha}$ consists of functions $f \in H(\mathbb{D})$ such that

$$
\|f\|_{\mathscr{A}_{\infty}^{\alpha}}=\sup _{z \in \mathbb{D}}\left(1-|z|^{2}\right)^{\alpha}|f(z)|<\infty .
$$

For information of such spaces, see, for example, [1-4].
For $a \in \mathbb{D}$, let $\sigma_{a}(z)=(a-z) /(1-\bar{a} z)$ be the automorphism of $\mathbb{D}$ that interchanges 0 and $a$. Let the Green function in $\mathbb{D}$ with logarithmic singularity at $a$ be given by

$$
g(z, a)=\log \left|\frac{1-\bar{a} z}{a-z}\right|=\log \frac{1}{\left|\sigma_{a}(z)\right|} .
$$

The space BMOA consists of all $f$ in the Hardy space $H^{2}$ such that

$$
\sup _{a \in \mathbb{D}}\left\|f \circ \sigma_{a}-f(a)\right\|_{H^{2}}<\infty .
$$

BMOA is a Banach space under following norm (see, e.g., [5]):

$$
\|f\|_{\text {BMOA }}=|f(0)|+\sup _{a \in \mathbb{D}}\left\|f \circ \sigma_{a}-f(a)\right\|_{H^{2}} .
$$

Let $\varphi$ and $\psi$ be holomorphic maps on the open unit disk $\mathbb{D}$ such that $\varphi(\mathbb{D}) \subset \mathbb{D}$. For a nonnegative integer $n$, we define a linear operator $D_{\varphi, \psi}^{n}$ as follows:

$$
D_{\varphi, \psi}^{n} f=\psi \cdot\left(f^{(n)} \circ \varphi\right), \quad f \in H(\mathbb{D}) .
$$

We call it weighted differentiation composition operators, which was defined in $[6,7]$. If $n=0$ and $\psi \equiv 1, D_{\varphi, \psi}^{n}$ becomes $C_{\varphi}$ induced by $\varphi$, defined as $C_{\varphi} f=f \circ \varphi, f \in H(\mathbb{D})$. If $\psi=$ 1 and $\varphi(z)=z$, then $D_{\varphi, \psi}^{n}$ is the differentiation operator defined as $D^{n} f=f^{(n)}$. If $n=0$, then we get the weighted 
composition operator $\psi C_{\varphi}$ defined as $\psi C_{\varphi} f=\psi \cdot(f \circ \varphi)$. If $n=1$ and $\psi(z)=\varphi^{\prime}(z)$, then $D_{\varphi, \psi}^{n}$ reduces to $D C_{\varphi}$. When $\psi \equiv$ 1 , then $D_{\varphi, \psi}^{n}$ reduces to differentiation composition operator $C_{\varphi} D^{n}$ (also named as product of differentiation and composition operator). If we put $\varphi(z)=z$, then $D_{\varphi, \psi}^{n}=M_{\psi} D^{n}$, the product of multiplication and differentiation operator.

The boundedness and compactness of differentiation composition operator between spaces of holomorphic functions have been studied extensively. For example, Hibschweiler; Portnoy and Ohno studied differentiation composition operator $C_{\varphi} D$ on Hardy and Bergman spaces in $[8,9]$; Li; Stević and Ohno studied $C_{\varphi} D$ on Bloch type spaces in [10-12]; Wu and Wulan gave a new compactness criterion of $C_{\varphi} D^{m}$ on the Bloch space in [13]. Recently, the weighted differentiation composition operator between different function spaces has also been investigated by several authors (see, for example, [14-21]).

Boundedness, compactness, and essential norm of weighted composition operator $\psi C_{\varphi}$ between Bloch-type spaces have been studied in [22-24]. Recently, Manhas and Zhao [25] and Hyvärinen and Lindström [26] gave a new characterization of boundedness and compactness of $\psi C_{\varphi}$ in terms of the norm of $\varphi^{n}$ (for the compactness of composition operator, see $[27,28])$.

Motivated by $[13,25,26]$, we study the operator $D_{\varphi, \psi}^{n}(n \geq$ 1) from BMOA and Bloch space to Bloch-type spaces.

Throughout this paper, constants are denoted by $C$; they are positive and not necessarily the same at each occurrence. The notation $A \lesssim B$ means that there is a positive constant $C$ such that $A \leq C B$. When $A \lesssim B$ and $B \lesssim A$, we write $A \approx B$.

\section{Some Lemmas}

It is well known that $H^{\infty} \subset \mathrm{BMOA} \subset \mathscr{B}$. From the definition of the norm, we know

$$
\|f\|_{\text {BMOA }} \leqslant\|f\|_{\infty}, \quad f \in H^{\infty} .
$$

Indeed, Girela proved that

$$
\|f\|_{\mathscr{B}} \leq\|f\|_{\mathrm{BMOA}_{1}}
$$

in Corollary 5.2 of [5]. The following lemma is from Lemma 5 in [29] (see also Lemma 4.12 of [4]).

Lemma 1. If $f \in H(\mathbb{D})$, then

$$
|f(0)|^{2} \leq 2 \int_{\mathbb{D}}|f(z)|^{2} \log \frac{1}{|z|} d A(z) .
$$

The following lemma may be known, but we fail to find its reference; so we give a proof for the completeness of the paper.

Lemma 2. Let $f \in H(\mathbb{D})$. Then,

$$
\|f\|_{\mathscr{B}} \leq\|f\|_{B M O A} .
$$

Proof. Applying Littlewood-Paley identity

$$
\|f\|_{H^{2}}^{2}=|f(0)|^{2}+2 \int_{\mathbb{D}}\left|f^{\prime}(z)\right|^{2} \log \frac{1}{|z|} d A(z)
$$

and Lemma 1, we have

$$
\begin{aligned}
\sup _{a \in \mathbb{D}}\left\|f \circ \sigma_{a}-f(a)\right\|_{H^{2}} \\
=\sup _{a \in \mathbb{D}}\left(2 \int_{\mathbb{D}}\left|f^{\prime}\left(\sigma_{a}(z)\right) \sigma_{a}^{\prime}(z)\right|^{2} \log \frac{1}{|z|} d A(z)\right)^{1 / 2} \\
\geq \sup _{a \in \mathbb{D}}\left(1-|a|^{2}\right)\left|f^{\prime}(a)\right| .
\end{aligned}
$$

It follows from the definitions of Bloch space and BMOA space that

$$
\|f\|_{\mathscr{B}} \leq\|f\|_{\mathrm{BMOA}} .
$$

By Theorem 6.2 of [5] and the proof of Theorem 1 of [30], we have the following lemma.

Lemma 3. Let $n$ be a fixed positive integer and $f \in \mathscr{B}$ with $f(0)=f^{\prime}(0)=\cdots=f^{(n-1)}(0)=0$. If

$$
\sup _{a \in \mathbb{D}} \int_{\mathbb{D}}\left|f^{(n)}(z)\right|^{2}\left(1-|z|^{2}\right)^{2 n-2}\left(1-\left|\sigma_{a}(z)\right|^{2}\right) d A(z) \lesssim 1,
$$

then $\|f\|_{B M O A} \leqslant 1$.

Lemma 4. Suppose that $n$ is a fixed positive integer. Let $k \in$ $\mathbb{N}^{+}, 0 \leq x \leq 1$, and

$$
H_{k}^{n}(x)= \begin{cases}k(k-1) \cdots(k-n+1)(1-x)^{n} x^{k-n} & \text { if } k>n \\ n !(1-x)^{n} & \text { if } k=n .\end{cases}
$$

If $k \geq n$, then there are two positive constants $c_{n}$ and $C_{n}$, depending only on $n$, such that

$$
c_{n} \leq H_{k}^{n}(x) \leq C_{n}, \quad \text { for } \frac{k-n}{k} \leq x \leq \frac{k-n+1}{k+1} .
$$

Proof. The proof is similar to that of Lemma 2.2 of [13] and is so omitted.

\section{Boundedness of $D_{\varphi, \psi}^{n}$}

In this section, we characterize the boundedness of $D_{\varphi, \psi}^{n}$ from BMOA and the Bloch space to Bloch-type spaces.

Theorem 5. Let $\alpha>0, \psi \in H(\mathbb{D}), n \in \mathbb{N}^{+}$, and $\varphi$ a holomorphic self-map of $\mathbb{D}$. Then, the following statements are equivalent:
(a) $D_{\varphi, \psi}^{n}: B M O A \rightarrow \mathscr{B}^{\alpha}$ is bounded.
(b) $D_{\varphi, \psi^{\prime}}^{n}: B M O A \rightarrow \mathscr{A}_{\infty}^{\alpha}$ and $D_{\varphi, \psi \varphi^{\prime}}^{n+1}: B M O A \rightarrow \mathscr{A}_{\infty}^{\alpha}$ are bounded.
(c) $D_{\varphi, \psi}^{n}: \mathscr{B}_{0} \rightarrow \mathscr{B}^{\alpha}$ is bounded. 
(d) $D_{\varphi, \psi^{\prime}}^{n}: \mathscr{B}_{0} \rightarrow \mathscr{A}_{\infty}^{\alpha}$ and $D_{\varphi, \psi \varphi^{\prime}}^{n+1}: \mathscr{B}_{0} \rightarrow \mathscr{A}_{\infty}^{\alpha}$ are bounded.

(e) $D_{\varphi, \psi}^{n}: \mathscr{B} \rightarrow \mathscr{B}^{\alpha}$ is bounded.

(f) $D_{\varphi, \psi^{\prime}}^{n}: \mathscr{B} \rightarrow \mathscr{A}_{\infty}^{\alpha}$ and $D_{\varphi, \psi \varphi^{\prime}}^{n+1}: \mathscr{B} \rightarrow \mathscr{A}_{\infty}^{\alpha}$ are bounded.

(g) $\sup _{z \in \mathbb{D}}\left(\left(1-|z|^{2}\right)^{\alpha} /\left(1-|\varphi(z)|^{2}\right)^{n}\right)\left|\psi^{\prime}(z)\right|<\infty$ and $\sup _{z \in \mathbb{D}}\left(\left(1-|z|^{2}\right)^{\alpha} /\left(1-|\varphi(z)|^{2}\right)^{n+1}\right)\left|\psi(z) \varphi^{\prime}(z)\right|<\infty$.

(h) $\sup _{k \in \mathbb{N}}\left\|D_{\varphi, \psi^{\prime}}^{n}\left(z^{k}\right)\right\|_{\mathscr{A}_{\infty}^{\alpha}}<\infty$ and $\sup _{k \in \mathbb{N}}\left\|D_{\varphi, \psi \varphi^{\prime}}^{n+1}\left(z^{k}\right)\right\|_{\mathscr{H}_{\infty}^{\alpha}}<\infty$.

Proof. It is obvious that (f) $\Rightarrow$ (b), (f) $\Rightarrow$ (d), (e) $\Rightarrow$ (c), and (e) $\Rightarrow$ (a). Thus, we will prove the theorem according to the following steps. (I): (a) $\Rightarrow$ (g), (c) $\Rightarrow$ (g). (II): (b) $\Rightarrow$ (g), (d) $\Rightarrow$ (g). (III): (g) $\Rightarrow$ (e), (g) $\Rightarrow$ (f). (IV): (f) $\Leftrightarrow$ (h).

(I): (a) $\Rightarrow(\mathrm{g}),(\mathrm{c}) \Rightarrow(\mathrm{g})$. Suppose that (a) or (c) holds. We choose the test function $g_{1}(z)=z^{n}$. By Lemma 2 , we get

$$
\left\|g_{1}\right\|_{\mathscr{B}} \leq\left\|g_{1}\right\|_{\mathrm{BMOA}} \leqslant\left\|g_{1}\right\|_{\infty}=1 .
$$

So

$$
\sup _{z \in \mathbb{D}}\left(1-|z|^{2}\right)^{\alpha}\left|\psi^{\prime}(z)\right| \leq\left\|D_{\varphi, \psi}^{n} g_{1}\right\|_{\mathscr{B}^{\alpha}}<\infty .
$$

Taking $g_{2}(z)=z^{n+1}$ and using the fact that $|\varphi(z)|<1$, we have

$$
\begin{aligned}
\sup _{z \in \mathbb{D}}(1 & \left.-|z|^{2}\right)^{\alpha}\left|\psi(z) \varphi^{\prime}(z)\right| \\
& \leq\left\|D_{\varphi, \psi}^{n} g_{2}\right\|_{\mathscr{B}^{\alpha}}+\left\|D_{\varphi, \psi}^{n} g_{1}\right\|_{\mathscr{B}^{\alpha}}<\infty .
\end{aligned}
$$

We now consider the function

$$
f_{\lambda}(z)=(n+1) \frac{1-|\varphi(\lambda)|^{2}}{1-\overline{\varphi(\lambda)} z}-\frac{\left(1-|\varphi(\lambda)|^{2}\right)^{2}}{(1-\overline{\varphi(\lambda)} z)^{2}}, \quad \lambda \in \mathbb{D} .
$$

It is easy to check that $f_{\lambda} \in \mathscr{B}_{0} \cap \mathrm{BMOA}$ and $\left\|f_{\lambda}\right\|_{\mathrm{BMOA}} \lesssim$ $\left\|f_{\lambda}\right\|_{\infty} \lesssim 1$. Moreover,

$$
\begin{aligned}
f_{\lambda}^{(n)}(z)= & (n+1) !(\overline{\varphi(\lambda)})^{n} \\
& \times\left[\frac{1-|\varphi(\lambda)|^{2}}{(1-\overline{\varphi(\lambda)} z)^{n+1}}-\frac{\left(1-|\varphi(\lambda)|^{2}\right)^{2}}{(1-\overline{\varphi(\lambda)} z)^{n+2}}\right] .
\end{aligned}
$$

Thus, $f_{\lambda}^{(n)}(\varphi(\lambda))=0$ and

$$
f_{\lambda}^{(n+1)}(\varphi(\lambda))=\frac{-(n+1) !(\overline{\varphi(\lambda)})^{n+1}}{\left(1-|\varphi(\lambda)|^{2}\right)^{n+1}} .
$$

We obtain

$$
\begin{aligned}
& \left\|D_{\varphi, \psi}^{n}\right\| \gtrsim\left\|D_{\varphi, \psi}^{n} f_{\lambda}\right\|_{\mathscr{B}^{\alpha}} \\
& z\left(1-|\lambda|^{2}\right)^{\alpha} \mid \psi^{\prime}(\lambda) f_{\lambda}^{(n)}(\varphi(\lambda)) \\
& +\psi(\lambda) \varphi^{\prime}(\lambda) f_{\lambda}^{(n+1)}(\varphi(\lambda)) \\
& \gtrsim(n+1) ! \frac{\left(1-|\lambda|^{2}\right)^{\alpha}}{\left(1-|\varphi(\lambda)|^{2}\right)^{n+1}}|\varphi(\lambda)|^{n+1}\left|\psi(\lambda) \varphi^{\prime}(\lambda)\right| .
\end{aligned}
$$

Thus, for any $r_{0} \in(0,1)$, we have

$$
\sup _{r_{0}<|\varphi(\lambda)|<1} \frac{\left(1-|\lambda|^{2}\right)^{\alpha}}{\left(1-|\varphi(\lambda)|^{2}\right)^{n+1}}\left|\psi(\lambda) \varphi^{\prime}(\lambda)\right|<\infty .
$$

Using (21) yields

$$
\begin{aligned}
& \sup _{|\varphi(\lambda)| \leq r_{0}} \frac{\left(1-|\lambda|^{2}\right)^{\alpha}}{\left(1-|\varphi(\lambda)|^{2}\right)^{n+1}}\left|\psi(\lambda) \varphi^{\prime}(\lambda)\right| \\
& \quad \lesssim \frac{1}{\left(1-r_{0}^{2}\right)^{n+1}} \sup _{\lambda \in \mathbb{D}}\left(1-|\lambda|^{2}\right)^{\alpha}\left|\psi(\lambda) \varphi^{\prime}(\lambda)\right| \\
& \quad<\infty .
\end{aligned}
$$

Combining (26) with (27), we get

$$
\sup _{\lambda \in \mathbb{D}} \frac{\left(1-|\lambda|^{2}\right)^{\alpha}}{\left(1-|\varphi(\lambda)|^{2}\right)^{n+1}}\left|\psi(\lambda) \varphi^{\prime}(\lambda)\right|<\infty .
$$

We next consider the function

$$
g_{\lambda}(z)=(n+2) \frac{1-|\varphi(\lambda)|^{2}}{1-\overline{\varphi(\lambda)} z}-\frac{\left(1-|\varphi(\lambda)|^{2}\right)^{2}}{(1-\overline{\varphi(\lambda)} z)^{2}}, \quad \lambda \in \mathbb{D} .
$$

Similarly, we get $g_{\lambda} \in \mathscr{B}_{0} \cap \mathrm{BMOA}$ and

$$
\left\|g_{\lambda}\right\|_{\mathrm{BMOA}} \lesssim\left\|g_{\lambda}\right\|_{\infty} \lesssim 1 \text {. }
$$

Moreover,

$$
\begin{aligned}
g_{\lambda}^{(n)}(z)=n !(\overline{\varphi(\lambda)})^{n}[ & (n+2) \frac{1-|\varphi(\lambda)|^{2}}{(1-\overline{\varphi(\lambda)} z)^{n+1}} \\
& \left.-(n+1) \frac{\left(1-|\varphi(\lambda)|^{2}\right)^{2}}{(1-\overline{\varphi(\lambda)} z)^{n+2}}\right] .
\end{aligned}
$$

So

$$
g_{\lambda}^{(n)}(\varphi(\lambda))=\frac{n !(\overline{\varphi(\lambda)})^{n}}{\left(1-|\varphi(\lambda)|^{2}\right)^{n}}
$$


and $g_{\lambda}^{(n+1)}(\varphi(\lambda))=0$. We have, as above,

$$
\begin{aligned}
\left\|D_{\varphi, \psi}^{n}\right\| & \geqslant\left\|D_{\varphi, \psi}^{n} g_{\lambda}\right\|_{\mathscr{B}^{\alpha}} \\
& \geqslant n ! \frac{\left(1-|\lambda|^{2}\right)^{\alpha}}{\left(1-|\varphi(\lambda)|^{2}\right)^{n}}|\varphi(\lambda)|^{n}\left|\psi^{\prime}(\lambda)\right| .
\end{aligned}
$$

Thus, for any $s_{0} \in(0,1)$,

$$
\sup _{s_{0}<|\varphi(\lambda)|<1} \frac{\left(1-|\lambda|^{2}\right)^{\alpha}}{\left(1-|\varphi(\lambda)|^{2}\right)^{n}}\left|\psi^{\prime}(\lambda)\right|<\infty .
$$

Applying (20), we get

$$
\sup _{|\varphi(\lambda)| \leq s_{0}} \frac{\left(1-|\lambda|^{2}\right)^{\alpha}}{\left(1-|\varphi(\lambda)|^{2}\right)^{n}}\left|\psi^{\prime}(\lambda)\right|<\infty .
$$

Combining (34) with (35) yields

$$
\sup _{\lambda \in \mathbb{D}} \frac{\left(1-|\lambda|^{2}\right)^{\alpha}}{\left(1-|\varphi(\lambda)|^{2}\right)^{n}}\left|\psi^{\prime}(\lambda)\right|<\infty
$$

(II): (b) $\Rightarrow$ (g) and (d) $\Rightarrow$ (g). Suppose that $D_{\varphi, \psi^{\prime}}^{n} \quad$ : $\mathrm{BMOA} \rightarrow \mathscr{A}_{\infty}^{\alpha}$ is bounded or $D_{\varphi, \psi^{\prime}}^{n}: \mathscr{B}_{0} \rightarrow \mathscr{A}_{\infty}^{\alpha}$ is bounded. Set

$$
\lambda=\sup _{z \in \mathbb{D}} \frac{\left(1-|z|^{2}\right)^{\alpha}}{\left(1-|\varphi(z)|^{2}\right)^{n}}\left|\psi^{\prime}(z)\right| .
$$

If $\lambda=\infty$, then for any positive integer $N$, we can find $b \in \mathbb{D}$ such that

$$
\frac{\left(1-|b|^{2}\right)^{\alpha}}{\left(1-|\varphi(b)|^{2}\right)^{n}}\left|\psi^{\prime}(b)\right|>N
$$

If $\varphi(b)=0$, then choose the test function $g(z)=z^{n}$. It is clear that $g \in \mathscr{B}_{0}$. From Lemma 2, we have

$$
\|g\|_{\mathscr{B}} \leq\|g\|_{\mathrm{BMOA}} \leqslant\|g\|_{\infty}=1 .
$$

So

$$
\left\|D_{\varphi, \psi^{\prime}}^{n}\right\| \geq\left\|D_{\varphi, \psi^{\prime}}^{n} g\right\|_{\mathscr{A}_{\infty}^{\alpha}}>\left(1-|b|^{2}\right)^{\alpha}\left|\psi^{\prime}(b)\right|>N .
$$

If $\varphi(b) \neq 0$, consider the function

$$
g(z)=\frac{1}{\bar{a}^{n}} \frac{\left(1-|a|^{2}\right)^{n}}{(1-\bar{a} z)^{n}} \triangleq \sum_{j=0}^{\infty} c_{j} z^{j}
$$

where $a=\varphi(b)$. Let $F(z)=\sum_{j=n}^{\infty} c_{j} z^{j}$. Then, $F(0)=F^{\prime}(0)=$ $\cdots=F^{(n-1)}(0)=0$ and

$$
F^{(n)}(z)=\left(\frac{1-|a|^{2}}{(1-\bar{a} z)^{2}}\right)^{n} .
$$

It is easy to see that

$$
\left(1-|z|^{2}\right)^{n}\left|F^{(n)}(z)\right|=\left(1-\left|\sigma_{a}(z)\right|^{2}\right)^{n} \leq 1 .
$$

So, by Theorems 5.4 and 5.13 of [4], we have $F \in \mathscr{B}_{0}$ and $\|F\|_{\mathscr{B}} \lesssim 1$. By Lemma 1 of [31] and Lemma 3, we get $\|F\|_{\mathrm{BMOA}} \lesssim 1$. We have

$$
\left\|D_{\varphi, \psi^{\prime}}^{n}\right\| \gtrsim\left\|D_{\varphi, \psi^{\prime}}^{n} F\right\|_{\mathscr{A}_{\infty}^{\alpha}}>\frac{\left(1-|b|^{2}\right)^{\alpha}}{\left(1-|\varphi(b)|^{2}\right)^{n}}\left|\psi^{\prime}(b)\right|>N .
$$

Since $N$ is arbitrary, we get $\left\|D_{\varphi, \psi^{\prime}}^{n}\right\|=\infty$. This contradicts the boundedness of $D_{\varphi, \psi^{\prime}}^{n}: \mathrm{BMOA} \rightarrow \mathscr{A}_{\infty}^{\alpha}$ and that of $D_{\varphi, \psi^{\prime}}^{n}:$ $\mathscr{B}_{0} \rightarrow \mathscr{A}_{\infty}^{\alpha}$.

Now, suppose that $D_{\varphi, \psi \varphi^{\prime}}^{n+1}:$ BMOA $\rightarrow \mathscr{A}_{\infty}^{\alpha}$ is bounded or $D_{\varphi, \psi \varphi^{\prime}}^{n+1}: \mathscr{B}_{0} \rightarrow \mathscr{A}_{\infty}^{\alpha}$ is bounded. Set

$$
\eta=\sup _{z \in \mathbb{D}} \frac{\left(1-|z|^{2}\right)^{\alpha}}{\left(1-|\varphi(z)|^{2}\right)^{n+1}}\left|\psi(z) \varphi^{\prime}(z)\right| .
$$

If $\eta=\infty$, then for any positive integer $M$, exists $u \in \mathbb{D}$ such that

$$
\frac{\left(1-|u|^{2}\right)^{\alpha}}{\left(1-|\varphi(u)|^{2}\right)^{n+1}}\left|\psi(u) \varphi^{\prime}(u)\right|>M .
$$

If $\varphi(u)=0$, then set $g(z)=z^{n+1}$. The process as above gives

$$
\left\|D_{\varphi, \psi \varphi^{\prime}}^{n+1}\right\| \gtrsim\left\|D_{\varphi, \psi \varphi^{\prime}}^{n+1} g\right\|_{\mathscr{A}_{\infty}^{\alpha}}>M .
$$

If $\varphi(u) \neq 0$, consider the function

$$
g(z)=\frac{1}{\bar{a}^{n+1}} \frac{\left(1-|a|^{2}\right)^{n+1}}{(1-\bar{a} z)^{n+1}} \triangleq \sum_{j=0}^{\infty} c_{j} z^{j},
$$

where $a=\varphi(u)$. Let $F(z)=\sum_{j=n+1}^{\infty} c_{j} z^{j}$. Then, $F(0)=F^{\prime}(0)=$ $\cdots=F^{(n)}(0)=0$ and

$$
F^{(n+1)}(z)=\left(\frac{1-|a|^{2}}{(1-\bar{a} z)^{2}}\right)^{n+1},
$$

$$
\left(1-|z|^{2}\right)^{n+1}\left|F^{(n+1)}(z)\right|=\left(1-\left|\sigma_{a}(z)\right|^{2}\right)^{n+1} \leq 1 .
$$

Applying Theorems 5.4 and 5.13 of [4] again yields $F \in \mathscr{B}_{0}$ and $\|F\|_{\mathscr{B}} \lesssim 1$. We get $\|F\|_{\mathrm{BMOA}} \lesssim 1$ and

$$
\begin{aligned}
\left\|D_{\varphi, \psi \varphi^{\prime}}^{n+1}\right\| & \gtrsim\left\|D_{\varphi, \psi \varphi^{\prime}}^{n+1} F\right\|_{\mathscr{A}_{\infty}^{\alpha}} \\
& >\frac{\left(1-|u|^{2}\right)^{\alpha}}{\left(1-|\varphi(u)|^{2}\right)^{n+1}}\left|\psi(u) \varphi^{\prime}(u)\right|>M .
\end{aligned}
$$

Since $M$ is arbitrary, we have $\left\|D_{\varphi, \psi \varphi^{\prime}}^{n+1}\right\|=\infty$. This contradicts the boundedness of $D_{\varphi, \psi \varphi^{\prime}}^{n+1}$. 


$$
\begin{aligned}
& \text { (III): (g) } \Rightarrow(\mathrm{e}),(\mathrm{g}) \Rightarrow \text { (f). Note that } \\
& \left\|D_{\varphi, \psi}^{n} f\right\|_{\mathscr{B}^{\alpha}}=\sup _{z \in \mathbb{D}}\left(1-|z|^{2}\right)^{\alpha} \\
& \times \mid \psi(z) \varphi^{\prime}(z) f^{(n+1)}(\varphi(z)) \\
& +\psi^{\prime}(z) f^{(n)}(\varphi(z)) \mid \\
& \leqslant\|f\|_{\mathscr{B}}\left[\sup _{z \in \mathbb{D}} \frac{\left(1-|z|^{2}\right)^{\alpha}}{\left(1-|\varphi(z)|^{2}\right)^{n+1}}\left|\psi(z) \varphi^{\prime}(z)\right|\right. \\
& \left.+\sup _{z \in \mathbb{D}} \frac{\left(1-|z|^{2}\right)^{\alpha}}{\left(1-|\varphi(z)|^{2}\right)^{n}}\left|\psi^{\prime}(z)\right|\right], \\
& \left\|D_{\varphi, \psi \varphi^{\prime}}^{n+1} f\right\|_{\mathscr{A}_{\infty}^{\alpha}}=\sup _{z \in \mathbb{D}}\left(1-|z|^{2}\right)^{\alpha} \\
& \times\left|\psi(z) \varphi^{\prime}(z) f^{(n+1)}(\varphi(z))\right| \\
& \lesssim\|f\|_{\mathscr{B}}\left[\sup _{z \in \mathbb{D}} \frac{\left(1-|z|^{2}\right)^{\alpha}}{\left(1-|\varphi(z)|^{2}\right)^{n+1}}\left|\psi(z) \varphi^{\prime}(z)\right|\right], \\
& \left\|D_{\varphi, \psi^{\prime}}^{n} f\right\|_{\mathcal{A}_{\infty}^{\alpha}}=\sup _{z \in \mathbb{D}}\left(1-|z|^{2}\right)^{\alpha}\left|\psi^{\prime}(z) f^{(n)}(\varphi(z))\right| \\
& \lesssim\|f\|_{\mathscr{B}}\left[\sup _{z \in \mathbb{D}} \frac{\left(1-|z|^{2}\right)^{\alpha}}{\left(1-|\varphi(z)|^{2}\right)^{n}}\left|\psi^{\prime}(z)\right|\right] .
\end{aligned}
$$

The desired results follow.

(IV): (f) $\Leftrightarrow(\mathrm{h})$. Suppose that (f) is true. It follows from Proposition 5.1 of [4] that $\left\|z^{k}\right\|_{\mathscr{B}} \leq\left\|z^{k}\right\|_{\infty}=1(k \in \mathbb{N})$. So,

$$
\begin{aligned}
\sup _{k \in \mathbb{N}}\left\|D_{\varphi, \psi^{\prime}}^{n}\left(z^{k}\right)\right\|_{\mathscr{A}_{\infty}^{\alpha}} \leq\left\|D_{\varphi, \psi^{\prime}}^{n}\right\|<\infty, \\
\sup _{k \in \mathbb{N}}\left\|D_{\varphi, \psi \varphi^{\prime}}^{n+1}\left(z^{k}\right)\right\|_{\mathscr{A}_{\infty}^{\alpha}} \leq\left\|D_{\varphi, \psi \varphi^{\prime}}^{n+1}\right\|<\infty .
\end{aligned}
$$

Conversely, assume that (h) is true. It is easy to see that

$$
\begin{aligned}
\sup _{z \in \mathbb{D}}\left(1-|z|^{2}\right)^{\alpha}\left|\psi^{\prime}(z)\right| & \leq\left\|D_{\varphi, \psi^{\prime}}^{n}\left(z^{n}\right)\right\|_{\mathscr{A}_{\infty}^{\alpha}} \\
& \leq \sup _{k \in \mathbb{N}}\left\|D_{\varphi, \psi^{\prime}}^{n}\left(z^{k}\right)\right\|_{\mathscr{A}_{\infty}^{\alpha}}<\infty, \\
\sup _{z \in \mathbb{D}}\left(1-|z|^{2}\right)^{\alpha}\left|\psi(z) \varphi^{\prime}(z)\right| & \leq\left\|D_{\varphi, \psi \varphi^{\prime}}^{n+1}\left(z^{n+1}\right)\right\|_{\mathscr{A}_{\infty}^{\alpha}} \\
& \leq \sup _{k \in \mathbb{N}}\left\|D_{\varphi, \psi \varphi^{\prime}}^{n+1}\left(z^{k}\right)\right\|_{\mathscr{A}_{\infty}^{\alpha}}<\infty .
\end{aligned}
$$

If $\|\varphi\|_{\infty}<1$, then

$$
\begin{aligned}
\sup _{z \in \mathbb{D}} & \frac{\left(1-|z|^{2}\right)^{\alpha}}{\left(1-|\varphi(z)|^{2}\right)^{n}}\left|\psi^{\prime}(z)\right| \\
& <\frac{1}{\left(1-\|\varphi\|_{\infty}\right)^{n}} \sup _{z \in \mathbb{D}}\left(1-|\varphi(z)|^{2}\right)^{\alpha}\left|\psi^{\prime}(z)\right|<\infty, \\
\sup _{z \in \mathbb{D}} \frac{\left(1-|z|^{2}\right)^{\alpha}\left|\psi(z) \varphi^{\prime}(z)\right|}{\left(1-|\varphi(z)|^{2}\right)^{n+1}} & \quad \frac{1}{\left(1-\|\varphi\|_{\infty}\right)^{n+1}} \sup _{z \in \mathbb{D}}\left(1-|\varphi(z)|^{2}\right)^{\alpha}\left|\psi(z) \varphi^{\prime}(z)\right|<\infty .
\end{aligned}
$$

Hence, $(\mathrm{g})$ is true. From $(\mathrm{g}) \Rightarrow(\mathrm{f})$, we obtain that (f) is also true.

From now on, we assume that $\|\varphi\|_{\infty}=1$. For any integer $k \geq n$, let

$$
\Delta_{k}^{n}=\left\{z \in \mathbb{D}: \frac{k-n}{k} \leq|\varphi(z)| \leq \frac{k-n+1}{k+1}\right\} .
$$

Let $m$ with $m \geq n$ be the smallest positive integer such that $\Delta_{m}^{n} \neq \varnothing$. Since $\Delta_{k}^{n}$ is not empty for every integer $k \geq m$ and $\mathbb{D}=\cup_{k=m}^{\infty} \Delta_{k}^{n}$. By Lemma 4 , for $f \in \mathscr{B}$,

$$
\begin{aligned}
\left\|D_{\varphi, \psi^{\prime}}^{n} f\right\|_{\mathscr{A}_{\infty}^{\alpha}} & \\
= & \sup _{z \in \mathbb{D}}\left(1-|z|^{2}\right)^{\alpha}\left|f^{(n)}(\varphi(z))\right|\left|\psi^{\prime}(z)\right| \\
= & \sup _{k \geq m z \sup _{k}^{n}}\left(1-|z|^{2}\right)^{\alpha}\left|f^{(n)}(\varphi(z)) \psi^{\prime}(z)\right| \\
= & \sup _{k \geq m z \in \Delta_{k}^{n}} \sup _{k}(1-|\varphi(z)|)^{n}\left|f^{(n)}(\varphi(z))\right| \\
& \times \frac{\left(1-|z|^{2}\right)^{\alpha}\left|\psi^{\prime}(z)\right|\left(H_{k}^{n}(|\varphi(z)|) /(1-|\varphi(z)|)^{n}\right)}{H_{k}^{n}(|\varphi(z)|)} \\
\lesssim & \frac{1}{c_{n}}\|f\|_{\mathscr{B}} \sup _{k \in \mathbb{N}}\left\|D_{\varphi, \psi^{\prime}}^{n}\left(z^{k}\right)\right\|_{\mathscr{A}_{\infty}^{\alpha}} .
\end{aligned}
$$

So, $D_{\varphi, \psi^{\prime}}^{n}: \mathscr{B} \rightarrow \mathscr{A}_{\mathrm{\infty}}^{\alpha}$ is bounded. Similar argument implies

$$
\begin{aligned}
\left\|D_{\varphi, \psi \varphi^{\prime}}^{n+1} f\right\|_{\mathscr{A}_{\infty}^{\alpha}}= & \sup _{k \geq m+1} \sup _{z \in \Delta_{k}^{n+1}}\left(1-|z|^{2}\right)^{\alpha}\left|f^{(n+1)}(\varphi(z))\right| \\
& \times\left|\psi(z) \varphi^{\prime}(z)\right| \\
\lesssim & \frac{1}{c_{n+1}}\|f\|_{\mathscr{B}} \sup _{k \in \mathbb{N}}\left\|D_{\varphi, \psi \varphi^{\prime}}^{n+1}\left(z^{k}\right)\right\|_{\mathscr{A}_{\infty}^{\alpha}} .
\end{aligned}
$$

Thus, $D_{\varphi, \psi \varphi^{\prime}}^{n+1}: \mathscr{B} \rightarrow \mathscr{A}_{\infty}^{\alpha}$ is bounded. Theorem 5 is proved. 


\section{Compactness of $D_{\varphi, \psi}^{n}$}

The following criterion for the compactness is a useful tool and it follows from standard arguments, for example, Proposition 3.11 of [32] or Lemma 2.10 of [33].

Lemma 6. Let $\alpha>0, n \in \mathbb{N}^{+}$, and $X=\mathscr{B}_{0}, \mathscr{B}$, or $B M O A$. Suppose that $\psi$ and $\varphi$ are in $H(\mathbb{D})$ such that $\varphi(\mathbb{D}) \subset \mathbb{D}$. Then, $D_{\varphi, \psi}^{n}: X \rightarrow \mathscr{B}^{\alpha}$ is compact if and only iffor any sequence $\left\{f_{m}\right\}$ in $X$ with $\sup _{m}\left\|f_{m}\right\|_{X}<\infty$, which converges to zero locally uniformly on $\mathbb{D}$; we have $\lim _{m \rightarrow \infty}\left\|D_{\varphi, \psi}^{n} f_{m}\right\|_{\mathscr{B}^{\alpha}}=0$.

We now give the compactness of $D_{\varphi, \psi}^{n}$ from BMOA and the Bloch space to Bloch-type spaces.

Theorem 7. Let $\alpha>0, \psi \in H(\mathbb{D}), n \in \mathbb{N}^{+}$, and $\varphi$ a holomorphic self-map of $\mathbb{D}$. Then, the following statements are equivalent:

(a) $D_{\varphi, \psi}^{n}: B M O A \rightarrow \mathscr{B}^{\alpha}$ is compact.

(b) $D_{\varphi, \psi^{\prime}}^{n}: B M O A \rightarrow \mathscr{A}_{\infty}^{\alpha}$ is compact and $D_{\varphi, \psi \varphi^{\prime}}^{n+1}:$ $B M O A \rightarrow \mathscr{A}_{\infty}^{\alpha}$ is compact.

(c) $D_{\varphi, \psi}^{n}: \mathscr{B}_{0} \rightarrow \mathscr{B}^{\alpha}$ is compact.

(d) $D_{\varphi, \psi^{\prime}}^{n}: \mathscr{B}_{0} \rightarrow \mathscr{A}_{\infty}^{\alpha}$ is compact and $D_{\varphi, \psi \varphi^{\prime}}^{n+1}: \mathscr{B}_{0} \rightarrow$ $\mathscr{A}_{\infty}^{\alpha}$ is compact.

(e) $D_{\varphi, \psi}^{n}: \mathscr{B} \rightarrow \mathscr{B}^{\alpha}$ is compact.

(f) $D_{\varphi, \psi^{\prime}}^{n}: \mathscr{B} \rightarrow \mathscr{A}_{\infty}^{\alpha}$ is compact and $D_{\varphi, \psi \varphi^{\prime}}^{n+1}: \mathscr{B} \rightarrow \mathscr{A}_{\infty}^{\alpha}$ is compact.

(g) $\psi \in \mathscr{B}^{\alpha}, \psi \varphi^{\prime} \in \mathscr{A}_{\infty}^{\alpha}$,

$$
\begin{gathered}
\lim _{|\varphi(z)| \rightarrow 1} \frac{\left(1-|z|^{2}\right)^{\alpha}}{\left(1-|\varphi(z)|^{2}\right)^{n}}\left|\psi^{\prime}(z)\right|=0, \\
\lim _{|\varphi(z)| \rightarrow 1} \frac{\left(1-|z|^{2}\right)^{\alpha}}{\left(1-|\varphi(z)|^{2}\right)^{n+1}}\left|\psi(z) \varphi^{\prime}(z)\right|=0 .
\end{gathered}
$$

(h) $\lim \sup _{k \rightarrow \infty}\left\|D_{\varphi, \psi^{\prime}}^{n}\left(z^{k}\right)\right\|_{\mathscr{A}_{\infty}^{\alpha}}=0$

$$
\text { and } \lim \sup _{k \rightarrow \infty}\left\|D_{\varphi, \psi \varphi^{\prime}}^{n+1}\left(z^{k}\right)\right\|_{\mathscr{A}_{\infty}^{\alpha}}=0 .
$$

Proof. The proof is a modification of that of Theorem 5; so we give a sketch of the proof. We will prove the theorem according to the following steps. (I): (a) $\Rightarrow$ (g), (c) $\Rightarrow$ (g). (II): (b) $\Rightarrow$ (g), (d) $\Rightarrow$ (g). (III): (g) $\Rightarrow$ (e), (g) $\Rightarrow$ (f). (IV): (f) $\Leftrightarrow(\mathrm{h})$.

(I): (a) $\Rightarrow(\mathrm{g}),(\mathrm{c}) \Rightarrow(\mathrm{g})$. Suppose that (a) or (c) holds. Then by Theorem 5 , we have

$$
\begin{gathered}
\sup _{z \in \mathbb{D}}\left(1-|z|^{2}\right)^{\alpha}\left|\psi^{\prime}(z)\right| \leq \sup _{z \in \mathbb{D}} \frac{\left(1-|z|^{2}\right)^{\alpha}\left|\psi^{\prime}(z)\right|}{\left(1-|\varphi(z)|^{2}\right)^{n}}<\infty, \\
\sup _{z \in \mathbb{D}}\left(1-|z|^{2}\right)^{\alpha}\left|\psi(z) \varphi^{\prime}(z)\right| \\
\leq \sup _{z \in \mathbb{D}} \frac{\left(1-|z|^{2}\right)^{\alpha}\left|\psi(z) \varphi^{\prime}(z)\right|}{\left(1-|\varphi(z)|^{2}\right)^{n+1}}<\infty .
\end{gathered}
$$

That is, $\psi \in \mathscr{B}^{\alpha}, \psi \varphi^{\prime} \in \mathscr{A}_{\infty}^{\alpha}$.

Let $\left\{z_{j}\right\}$ be a sequence in $\mathbb{D}$ such that $\left|\varphi\left(z_{j}\right)\right| \rightarrow 1$ as $j \rightarrow$ $\infty$. Now, we consider the function

$$
f_{j}(z)=(n+1) \frac{1-\left|\varphi\left(z_{j}\right)\right|^{2}}{1-\overline{\varphi\left(z_{j}\right)} z}-\frac{\left(1-\left|\varphi\left(z_{j}\right)\right|^{2}\right)^{2}}{\left(1-\overline{\varphi\left(z_{j}\right)} z\right)^{2}} .
$$

Simple computation shows that $f_{j} \in \mathscr{B}_{0} \cap \mathrm{BMOA}$ and

$$
\left\|f_{j}\right\|_{\text {BMOA }} \lesssim\left\|f_{j}\right\|_{\infty} \lesssim 1 \text {. }
$$

It is also easy to check that $f_{j} \rightarrow 0$ uniformly on compact subsets of $\mathbb{D}$ as $j \rightarrow \infty$. Moreover,

$$
\begin{aligned}
f_{j}^{(n)}(z)= & (n+1) !\left(\overline{\varphi\left(z_{j}\right)}\right)^{n} \\
& \times\left[\frac{1-\left|\varphi\left(z_{j}\right)\right|^{2}}{\left(1-\overline{\varphi\left(z_{j}\right)} z\right)^{n+1}}-\frac{\left(1-\left|\varphi\left(z_{j}\right)\right|^{2}\right)^{2}}{\left(1-\overline{\varphi\left(z_{j}\right)} z\right)^{n+2}}\right] .
\end{aligned}
$$

We have

$\left\|D_{\varphi, \psi}^{n} f_{j}\right\|_{\mathscr{B}^{\alpha}}$

$$
z(n+1) ! \frac{\left(1-\left|z_{j}\right|^{2}\right)^{\alpha}}{\left(1-\left|\varphi\left(z_{j}\right)\right|^{2}\right)^{n+1}}\left|\varphi\left(z_{j}\right)\right|^{n+1}\left|\psi\left(z_{j}\right) \varphi^{\prime}\left(z_{j}\right)\right| .
$$

By Lemma 6, we get

$$
\lim _{\left|\varphi\left(z_{j}\right)\right| \rightarrow 1} \frac{\left(1-\left|z_{j}\right|^{2}\right)^{\alpha}}{\left(1-\left|\varphi\left(z_{j}\right)\right|^{2}\right)^{n+1}}\left|\psi\left(z_{j}\right) \varphi^{\prime}\left(z_{j}\right)\right|=0 .
$$

We next consider the function

$$
g_{j}(z)=(n+2) \frac{1-\left|\varphi\left(z_{j}\right)\right|^{2}}{1-\overline{\varphi\left(z_{j}\right)} z}-\frac{\left(1-\left|\varphi\left(z_{j}\right)\right|^{2}\right)^{2}}{\left(1-\overline{\varphi\left(z_{j}\right)} z\right)^{2}} .
$$

Similarly, we get $g_{j} \in \mathscr{B}_{0} \cap \mathrm{BMOA}$ and

$$
\left\|g_{j}\right\|_{\mathrm{BMOA}} \lesssim\left\|g_{j}\right\|_{\infty} \lesssim 1 .
$$


It is easy to see that $g_{j}$ converges to zero uniformly on compact subsets of $\mathbb{D}$ as $j \rightarrow \infty$ and

$$
\begin{aligned}
g_{j}^{(n)}(z)= & n !\left(\overline{\varphi\left(z_{j}\right)}\right)^{n} \\
\times & {\left[(n+2) \frac{1-\left|\varphi\left(z_{j}\right)\right|^{2}}{\left(1-\overline{\varphi\left(z_{j}\right)} z\right)^{n+1}}\right.} \\
& \left.\quad-(n+1) \frac{\left(1-\left|\varphi\left(z_{j}\right)\right|^{2}\right)^{2}}{\left(1-\overline{\varphi\left(z_{j}\right)} z\right)^{n+2}}\right] .
\end{aligned}
$$

Thus,

$$
\left\|D_{\varphi, \psi}^{n} g_{j}\right\|_{\mathscr{B}^{\alpha}} \gtrsim n ! \frac{\left(1-\left|z_{j}\right|^{2}\right)^{\alpha}}{\left(1-\left|\varphi\left(z_{j}\right)\right|^{2}\right)^{n}}\left|\varphi\left(z_{j}\right)\right|^{n}\left|\psi^{\prime}\left(z_{j}\right)\right| .
$$

Applying Lemma 6 again, we have

$$
\lim _{\left|\varphi\left(z_{j}\right)\right| \rightarrow 1} \frac{\left(1-\left|z_{j}\right|^{2}\right)^{\alpha}}{\left(1-\left|\varphi\left(z_{j}\right)\right|^{2}\right)^{n}}\left|\psi^{\prime}\left(z_{j}\right)\right|=0
$$

Since $z_{j} \in \mathbb{D}$ is arbitrary, we proved that $(\mathrm{g})$ is true.

(II) (b) $\Rightarrow$ (g), (d) $\Rightarrow$ (g). Suppose that (b) or (d) holds. A similar argument to (I) shows that $\psi \in \mathscr{B}^{\alpha}, \psi \varphi^{\prime} \in \mathscr{A}_{\infty}^{\alpha}$. Now, suppose that the equations in $(\mathrm{g})$ are not true. Then, there exists a sequence $\left\{z_{j}\right\}$ in $\mathbb{D}$ and $\delta>0$ such that $\left|\varphi\left(z_{j}\right)\right| \rightarrow 1$ as $j \rightarrow \infty$ and

$$
\begin{gathered}
\frac{\left(1-\left|z_{j}\right|^{2}\right)^{\alpha}}{\left(1-\left|\varphi\left(z_{j}\right)\right|^{2}\right)^{n}}\left|\psi^{\prime}\left(z_{j}\right)\right|>\delta, \\
\frac{\left(1-\left|z_{j}\right|^{2}\right)^{\alpha}}{\left(1-\left|\varphi\left(z_{j}\right)\right|^{2}\right)^{n+1}}\left|\psi\left(z_{j}\right) \varphi^{\prime}\left(z_{j}\right)\right|>\delta .
\end{gathered}
$$

Choose a subsequence of $\left\{z_{j}\right\}$ if necessary and suppose that $\inf _{j}\left|\varphi\left(z_{j}\right)\right|>1 / 2$. Let

$$
f_{j}(z)=\frac{1-\left|\varphi\left(z_{j}\right)\right|^{2}}{1-\overline{\varphi\left(z_{j}\right)} z}, \quad z \in \mathbb{D} .
$$

Then, it is easy to check that $f_{j} \in \mathscr{B}_{0} \cap \mathrm{BMOA}, f_{j} \rightarrow 0$, uniformly on compact subsets of $\mathbb{D}$ and

$$
f_{j}^{(n)}(z)=n ! \frac{1-\left|\varphi\left(z_{j}\right)\right|^{2}}{\left(1-\overline{\varphi\left(z_{j}\right)} z\right)^{n+1}}\left(\overline{\varphi\left(z_{j}\right)}\right)^{n} .
$$

Thus,

$$
\begin{aligned}
\left\|D_{\varphi, \psi^{\prime}}^{n} f_{j}\right\|_{\mathscr{A}_{\infty}^{\alpha}} \geq & n ! \frac{\left(1-\left|z_{j}\right|^{2}\right)^{\alpha}}{\left(1-\left|\varphi\left(z_{j}\right)\right|^{2}\right)^{n}}\left|\varphi\left(z_{j}\right)\right|^{n}\left|\psi^{\prime}\left(z_{j}\right)\right|>\frac{n ! \delta}{2^{n}}, \\
\left\|D_{\varphi, \psi \varphi^{\prime}}^{n+1} f_{j}\right\|_{\mathscr{A}_{\infty}^{\alpha}} \geq & (n+1) ! \frac{\left(1-\left|z_{j}\right|^{2}\right)^{\alpha}}{\left(1-\left|\varphi\left(z_{j}\right)\right|^{2}\right)^{n+1}} \\
& \times\left|\varphi\left(z_{j}\right)\right|^{n+1}\left|\psi\left(z_{j}\right) \varphi^{\prime}\left(z_{j}\right)\right|>\frac{(n+1) ! \delta}{2^{n+1}} .
\end{aligned}
$$

Those contradict the compactness of $D_{\varphi, \psi^{\prime}}^{n}$ and $D_{\varphi, \psi \varphi^{\prime}}^{n+1}$.

(III) $(\mathrm{g}) \Rightarrow(\mathrm{e}),(\mathrm{g}) \Rightarrow$ (f). Let $\left\{f_{m}\right\}$ be a norm bounded sequence in $\mathscr{B}$ that converges to zero uniformly on compact subsets of $\mathbb{D}$. Let $M=\sup _{m}\left\|f_{m}\right\|_{\mathscr{B}}<\infty$. For $\varepsilon>0$, then there exists $r_{0} \in(0,1)$ such that for $|\varphi(z)|>r_{0}$, we have

$$
\begin{gathered}
\frac{\left(1-|z|^{2}\right)^{\alpha}}{\left(1-|\varphi(z)|^{2}\right)^{n}}\left|\psi^{\prime}(z)\right|<\varepsilon, \\
\frac{\left(1-|z|^{2}\right)^{\alpha}}{\left(1-|\varphi(z)|^{2}\right)^{n+1}}\left|\psi(z) \varphi^{\prime}(z)\right|<\varepsilon .
\end{gathered}
$$

Thus, for $z \in \mathbb{D}$, we have

$$
\begin{aligned}
\left\|D_{\varphi, \psi}^{n} f_{m}\right\|_{\mathscr{B}^{\alpha}} \lesssim & \left|\psi(0) f_{m}^{(n)}(\varphi(0))\right| \\
& +\sup _{|\varphi(z)| \leq r_{0}}\left(1-|z|^{2}\right)^{\alpha}\left|f_{m}^{(n)}(\varphi(z))\right|\left|\psi^{\prime}(z)\right| \\
& +\sup _{|\varphi(z)|>r_{0}} \frac{\left(1-|z|^{2}\right)^{\alpha}\left|\psi^{\prime}(z)\right|\left\|f_{m}\right\|_{\mathscr{B}}}{\left(1-|\varphi(z)|^{2}\right)^{n}} \\
& +\sup _{|\varphi(z)| \leq r_{0}}\left(1-|z|^{2}\right)^{\alpha}\left|f_{m}^{(n+1)}(\varphi(z))\right| \\
& \times\left|\psi(z) \varphi^{\prime}(z)\right| \\
& +\sup _{|\varphi(z)|>r_{0}} \frac{\left(1-|z|^{2}\right)^{\alpha}\left|\psi(z) \varphi^{\prime}(z)\right|\left\|f_{m}\right\|_{\mathscr{B}}}{\left(1-|\varphi(z)|^{2}\right)^{n+1}} \\
\leq & \left|\psi(0) f_{m}^{(n)}(\varphi(0))\right|+K_{1} \sup _{|z| \leq r_{0}}\left|f_{m}^{(n)}(z)\right| \\
& +K_{2} \sup _{|z| \leq r_{0}}\left|f_{m}^{(n+1)}(z)\right|+2 \varepsilon M,
\end{aligned}
$$

where $K_{1}=\sup _{z \in \mathbb{D}}\left(1-|z|^{2}\right)^{\alpha}\left|\psi^{\prime}(z)\right|$ and $K_{2}=\sup _{z \in \mathbb{D}}(1-$ $\left.|z|^{2}\right)^{\alpha}\left|\psi(z) \varphi^{\prime}(z)\right|$. Since $f_{m}^{(n)} \rightarrow 0$ uniformly on compact subsets of $\mathbb{D}$ as $m \rightarrow \infty$, we have $\left\|D_{\varphi, \psi}^{n} f_{m}\right\|_{\mathscr{B}^{\alpha}} \rightarrow 0$ as $m \rightarrow \infty$. It follows from Lemma 6 that $D_{\varphi, \psi}^{n}: \mathscr{B} \rightarrow \mathscr{B}^{\alpha}$ is compact. 
Similar as above, we know

$$
\begin{aligned}
\left\|D_{\varphi, \psi^{\prime}}^{n} f_{m}\right\|_{\mathscr{A}_{\infty}^{\alpha}} \lesssim & \sup _{|\varphi(z)| \leq r_{0}}\left(1-|z|^{2}\right)^{\alpha}\left|f_{m}^{(n)}(\varphi(z))\right|\left|\psi^{\prime}(z)\right| \\
& +\sup _{|\varphi(z)|>r_{0}} \frac{\left(1-|z|^{2}\right)^{\alpha}\left|\psi^{\prime}(z)\right|\left\|f_{m}\right\|_{\mathscr{B}}}{\left(1-|\varphi(z)|^{2}\right)^{n}} \\
\leq & K_{1} \sup _{|z| \leq r_{0}}\left|f_{m}^{(n)}(z)\right|+\varepsilon M, \\
\left\|D_{\varphi, \psi \varphi^{\prime}}^{n+1} f_{m}\right\|_{\mathscr{A}_{\infty}^{\alpha}} \leq & \sup _{|\varphi(z)| \leq r_{0}}\left(1-|z|^{2}\right)^{\alpha}\left|f_{m}^{(n+1)}(\varphi(z))\right| \\
& +\sup _{|\varphi(z)|>r_{0}} \frac{\left(1-|z|^{2}\right)^{\alpha}\left|\psi(z) \varphi^{\prime}(z)\right|\left\|f_{m}\right\|_{\mathscr{B}}}{\left(1-|\varphi(z)|^{2}\right)^{n+1}} \\
\leq & K_{2} \sup _{|z| \leq r_{0}}\left|f_{m}^{(n+1)}(z)\right|+\varepsilon M .
\end{aligned}
$$

From $f_{m}^{(n)} \rightarrow 0$ uniformly on compact subsets of $\mathbb{D}$, we have $\left\|D_{\varphi, \psi^{\prime}}^{n} f_{m}\right\|_{\mathscr{A}_{\infty}^{\alpha}} \rightarrow 0$ and $\left\|D_{\varphi, \psi \varphi^{\prime}}^{n+1} f_{m}\right\|_{\mathscr{A}_{\infty}^{\alpha}} \rightarrow 0$ as $m \rightarrow \infty$. So, $D_{\varphi, \psi^{\prime}}^{n}, D_{\varphi, \psi \varphi^{\prime}}^{n+1}: \mathscr{B} \rightarrow \mathscr{A}_{\infty}^{\alpha}$ are compact.

(IV): (f) $\Leftrightarrow(\mathrm{h})$. Suppose that (f) is true. Note that $\left\|z^{k}\right\|_{\mathscr{B}} \leq$ $\left\|z^{k}\right\|_{\infty}=1$ and $z^{k} \rightarrow 0$ uniformly on compact subsets of $\mathbb{D}$ as $k \rightarrow \infty$; by Lemma 6 , we have

$$
\begin{aligned}
& \lim _{k \rightarrow \infty}\left\|D_{\varphi, \psi^{\prime}}^{n}\left(z^{k}\right)\right\|_{\mathscr{A}_{\infty}^{\alpha}}=0, \\
& \lim _{k \rightarrow \infty}\left\|D_{\varphi, \psi \varphi^{\prime}}^{n+1}\left(z^{k}\right)\right\|_{\mathscr{A}_{\infty}^{\alpha}}=0 .
\end{aligned}
$$

Conversely, assume that $(\mathrm{h})$ is true. It is easy to see that

$$
\begin{aligned}
\sup _{z \in \mathbb{D}}\left(1-|z|^{2}\right)^{\alpha}\left|\psi^{\prime}(z)\right| \leq & \left\|D_{\varphi, \psi^{\prime}}^{n}\left(z^{n}\right)\right\|_{\mathscr{A}_{\infty}^{\alpha}} \\
& \leq \sup _{k \in \mathbb{N}}\left\|D_{\varphi, \psi^{\prime}}^{n}\left(z^{k}\right)\right\|_{\mathscr{A}_{\infty}^{\alpha}}<\infty, \\
\sup _{z \in \mathbb{D}}\left(1-|z|^{2}\right)^{\alpha}\left|\psi(z) \varphi^{\prime}(z)\right| & \leq\left\|D_{\varphi, \psi \varphi^{\prime}}^{n+1}\left(z^{n+1}\right)\right\|_{\mathscr{A}_{\infty}^{\alpha}} \\
& \leq \sup _{k \in \mathbb{N}}\left\|D_{\varphi, \psi \varphi^{\prime}}^{n+1}\left(z^{k}\right)\right\|_{\mathscr{A}_{\infty}^{\alpha}}<\infty .
\end{aligned}
$$

If $\|\varphi\|_{\infty}<1$, from (g) $\Rightarrow$ (f), we get that (f) is true. If $\|\varphi\|_{\infty}=$ 1 , as in the proof of Theorem 5 , let

$$
\Delta_{k}^{n}=\left\{z \in \mathbb{D}: \frac{k-n}{k} \leq|\varphi(z)| \leq \frac{k-n+1}{k+1}\right\} .
$$

And let $m$ with $m \geq n$ be the smallest positive integer such that $\Delta_{m}^{n} \neq \varnothing$. For given $\varepsilon>0$, there exists a large enough integer $M_{1}$ with $M_{1}>m$ such that

$$
\begin{gathered}
\left\|D_{\varphi, \psi^{\prime}}^{n}\left(z^{k}\right)\right\|_{\mathscr{A}_{\infty}^{\alpha}}<\varepsilon, \\
\left\|D_{\varphi, \psi \varphi^{\prime}}^{n+1}\left(z^{k}\right)\right\|_{\mathscr{A}_{\infty}^{\alpha}}<\varepsilon,
\end{gathered}
$$

whenever $k>M_{1}$. Let $\left\{f_{j}\right\}$ be a norm bounded sequence in $\mathscr{B}$ that converges to zero uniformly on compact subsets of $\mathbb{D}$ as $j \rightarrow \infty$. Denote $M=\sup _{m}\left\|f_{m}\right\|_{\mathscr{B}}<\infty$. We get

$$
\begin{aligned}
\| D_{\varphi, \psi^{\prime}}^{n} f_{j} & \|_{\mathscr{A}_{\infty}^{\alpha}} \\
= & \sup _{k \geq m z \in \Delta_{k}^{n}}\left(1-|z|^{2}\right)^{\alpha}\left|f_{j}^{(n)}(\varphi(z))\right|\left|\psi^{\prime}(z)\right| \\
= & \left(\sup _{m \leq k \leq M_{1}}+\sup _{k>M_{1}}\right) \sup _{z \in \Delta_{k}^{n}}\left(1-|z|^{2}\right)^{\alpha} \\
& \times\left|f_{j}^{(n)}(\varphi(z))\right|\left|\psi^{\prime}(z)\right| \\
= & : I_{1}+I_{2} .
\end{aligned}
$$

Then,

$$
\begin{aligned}
I_{1} & =\sup _{m \leq k \leq M_{1}} \sup _{z \in \Delta_{k}^{n}}\left(1-|z|^{2}\right)^{\alpha}\left|f_{j}^{(n)}(\varphi(z))\right|\left|\psi^{\prime}(z)\right| \\
& \leq \sup _{z \in \mathbb{D}}\left(1-|z|^{2}\right)^{\alpha}\left|\psi^{\prime}(z)\right| \sup _{|\varphi(z)| \leq r}\left|f_{j}^{(n)}(\varphi(z))\right|,
\end{aligned}
$$

where

$$
\begin{gathered}
r=\frac{M_{1}-n+1}{M_{1}+1} \\
I_{2}=\sup _{k \geq M_{1} z \in \Delta_{k}^{n}}\left(1-|z|^{2}\right)^{\alpha}\left|f_{j}^{(n)}(\varphi(z))\right|\left|\psi^{\prime}(z)\right| \\
=\sup _{k \geq M_{1} z \in \Delta_{k}^{n}}(1-|\varphi(z)|)^{n}\left|f_{j}^{(n)}(\varphi(z))\right| \\
\quad \times \frac{\left(1-|z|^{2}\right)^{\alpha}\left|\psi^{\prime}(z)\right|\left(H_{k}^{n}(|\varphi(z)|) /(1-|\varphi(z)|)^{n}\right)}{H_{k}^{n}(|\varphi(z)|)} \\
\lesssim \frac{1}{c_{n}}\left\|f_{j}\right\|_{\mathscr{B}} \sup _{k>M_{1}}\left\|D_{\varphi, \psi^{\prime}}^{n}\left(z^{k}\right)\right\|_{\mathscr{A}_{\infty}^{\alpha}} \\
\lesssim \frac{1}{c_{n}} \varepsilon .
\end{gathered}
$$

Since $f_{j}^{(n)} \rightarrow 0$ uniformly on compact subsets of $\mathbb{D}$, then $\left\|D_{\varphi, \psi^{\prime}}^{n} f_{j}\right\|_{\mathscr{A}_{\infty}^{\alpha}} \rightarrow 0$ as $j \rightarrow \infty$. Thus, by Lemma $6, D_{\varphi, \psi^{\prime}}^{n}:$ $\mathscr{B} \rightarrow \mathscr{A}_{\infty}^{\alpha}$ is compact. Similar as above, we can prove that $D_{\varphi, \psi \varphi^{\prime}}^{n+1}: \mathscr{B} \rightarrow \mathscr{A}_{\infty}^{\alpha}$ is compact. The proof is complete.

\section{Acknowledgments}

This work was supported by NNSF of China (Grant no. 11171203) and NSF of Guangdong Province (Grant nos. 10151503101000025 and S2011010004511). 


\section{References}

[1] P. L. Duren, Theory of $H^{p}$ Spaces, vol. 38 of Pure and Applied Mathematics, Academic Press, New York, NY, USA, 1970.

[2] H. T. Kaptanoğlu and S. Tülü, "Weighted Bloch, Lipschitz, Zygmund, Bers, and growth spaces of the ball: Bergman projections and characterizations," Taiwanese Journal of Mathematics, vol. 15, no. 1, pp. 101-127, 2011.

[3] K. Zhu, "Bloch type spaces of analytic functions," The Rocky Mountain Journal of Mathematics, vol. 23, no. 3, pp. 1143-1177, 1993.

[4] K. Zhu, Operator Theory in Function Spaces, vol. 138 of Mathematical Surveys and Monographs, American Mathematical Society, Providence, RI, USA, 2nd edition, 2007.

[5] D. Girela, "Analytic functions of bounded mean oscillation," in Complex Function Spaces (Mekrijärvi, 1999), vol. 4 of University of Joensuu, Department of Mathematics. Report Series, pp. 61170, University of Joensuu, Joensuu, Finland, 2001.

[6] X. Zhu, "Products of differentiation, composition and multiplication from Bergman type spaces to Bers type spaces," Integral Transforms and Special Functions, vol. 18, no. 3-4, pp. 223-231, 2007.

[7] X. Zhu, "Generalized weighted composition operators on weighted Bergman spaces," Numerical Functional Analysis and Optimization, vol. 30, no. 7-8, pp. 881-893, 2009.

[8] R. A. Hibschweiler and N. Portnoy, "Composition followed by differentiation between Bergman and Hardy spaces," The Rocky Mountain Journal of Mathematics, vol. 35, no. 3, pp. 843-855, 2005.

[9] S. Ohno, "Products of composition and differentiation between Hardy spaces," Bulletin of the Australian Mathematical Society, vol. 73, no. 2, pp. 235-243, 2006.

[10] S. Li and S. Stević, "Composition followed by differentiation between Bloch type spaces," Journal of Computational Analysis and Applications, vol. 9, no. 2, pp. 195-205, 2007.

[11] S. Ohno, "Products of differentiation and composition on Bloch spaces," Bulletin of the Korean Mathematical Society, vol. 46, no. 6, pp. 1135-1140, 2009.

[12] S. Stević, "Characterizations of composition followed by differentiation between Bloch-type spaces," Applied Mathematics and Computation, vol. 218, no. 8, pp. 4312-4316, 2011.

[13] Y. Wu and H. Wulan, "Products of differentiation and composition operators on the Bloch space," Collectanea Mathematica, vol. 63, no. 1, pp. 93-107, 2012.

[14] A. K. Sharma, "Generalized composition operators between weighted Bergman spaces," Acta Scientiarum Mathematicarum, vol. 78, pp. 187-211, 2012.

[15] A. Sharma and A. K. Sharma, "Carleson measures and a class of generalized integration operators on the Bergman space," The Rocky Mountain Journal of Mathematics, vol. 41, no. 5, pp. 17111724, 2011.

[16] A. K. Sharma, "Products of multiplication, composition and differentiation between weighted Bergman-Nevanlinna and Bloch-type spaces," Turkish Journal of Mathematics, vol. 35, no. 2, pp. 275-291, 2011.

[17] S. Stević, "Weighted differentiation composition operators from mixed-norm spaces to weighted-type spaces," Applied Mathematics and Computation, vol. 211, no. 1, pp. 222-233, 2009.

[18] S. Stević, "Weighted differentiation composition operators from $H^{\infty}$ and Bloch spaces to $n$th weighted-type spaces on the unit disk," Applied Mathematics and Computation, vol. 216, no. 12, pp. 3634-3641, 2010.
[19] S. Stević and A. K. Sharma, "Iterated differentiation followed by composition from Bloch-type spaces to weighted BMOA spaces," Applied Mathematics and Computation, vol. 218, no. 7, pp. 3574-3580, 2011.

[20] S. Stević and A. K. Sharma, "Composition operators from weighted Bergman-Privalov spaces to Zygmund type spaces on the unit disk," Annales Polonici Mathematici, vol. 105, no. 1, pp. 77-86, 2012.

[21] Y. Yu and Y. Liu, "Weighted differentiation composition operators from $H^{\infty}$ to Zygmund spaces," Integral Transforms and Special Functions, vol. 22, no. 7, pp. 507-520, 2011.

[22] B. D. MacCluer and R. Zhao, "Essential norms of weighted composition operators between Bloch-type spaces," The Rocky Mountain Journal of Mathematics, vol. 33, no. 4, pp. 1437-1458, 2003.

[23] A. Montes-Rodríguez, "Weighted composition operators on weighted Banach spaces of analytic functions," Journal of the London Mathematical Society, vol. 61, no. 3, pp. 872-884, 2000.

[24] S. Ohno, K. Stroethoff, and R. Zhao, "Weighted composition operators between Bloch-type spaces," The Rocky Mountain Journal of Mathematics, vol. 33, no. 1, pp. 191-215, 2003.

[25] J. S. Manhas and R. Zhao, "New estimates of essential norms of weighted composition operators between Bloch type spaces," Journal of Mathematical Analysis and Applications, vol. 389, no. 1, pp. 32-47, 2012.

[26] O. Hyvärinen and M. Lindström, "Estimates of essential norms of weighted composition operators between Bloch-type spaces," Journal of Mathematical Analysis and Applications, vol. 393, no. 1, pp. 38-44, 2012.

[27] H. Wulan, D. Zheng, and K. Zhu, "Compact composition operators on BMOA and the Bloch space," Proceedings of the American Mathematical Society, vol. 137, no. 11, pp. 3861-3868, 2009.

[28] R. Zhao, "Essential norms of composition operators between Bloch type spaces," Proceedings of the American Mathematical Society, vol. 138, no. 7, pp. 2537-2546, 2010.

[29] J. Liu and C. Xiong, "Norm-attaining integral operators on analytic function spaces," Journal of Mathematical Analysis and Applications, vol. 399, no. 1, pp. 108-115, 2013.

[30] R. Aulaskari, M. Nowak, and R. Zhao, “The $n$th derivative characterisation of Möbius invariant Dirichlet space," Bulletin of the Australian Mathematical Society, vol. 58, no. 1, pp. 43-56, 1998.

[31] R. Zhao, "Distances from Bloch functions to some Möbius invariant spaces," Annales Academiae Scientiarum Fennicae, vol. 33, no. 1, pp. 303-313, 2008.

[32] C. C. Cowen and B. D. MacCluer, Composition Operators on Spaces of Analytic Functions, Studies in Advanced Mathematics, CRC Press, Boca Raton, Fla, USA, 1995.

[33] M. Tjani, Compact composition operators on some Möbius invariant Banach spaces [Ph.D. thesis], Michigan State University, 1996. 


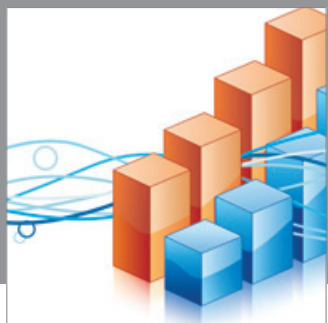

Advances in

Operations Research

mansans

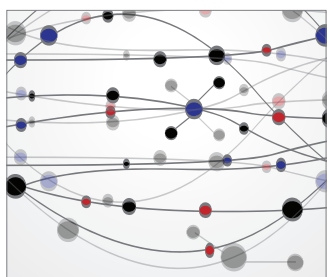

The Scientific World Journal
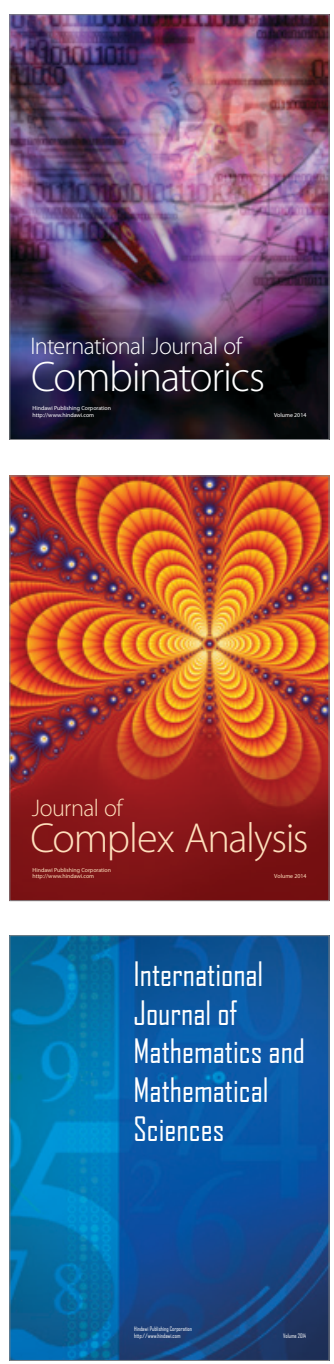
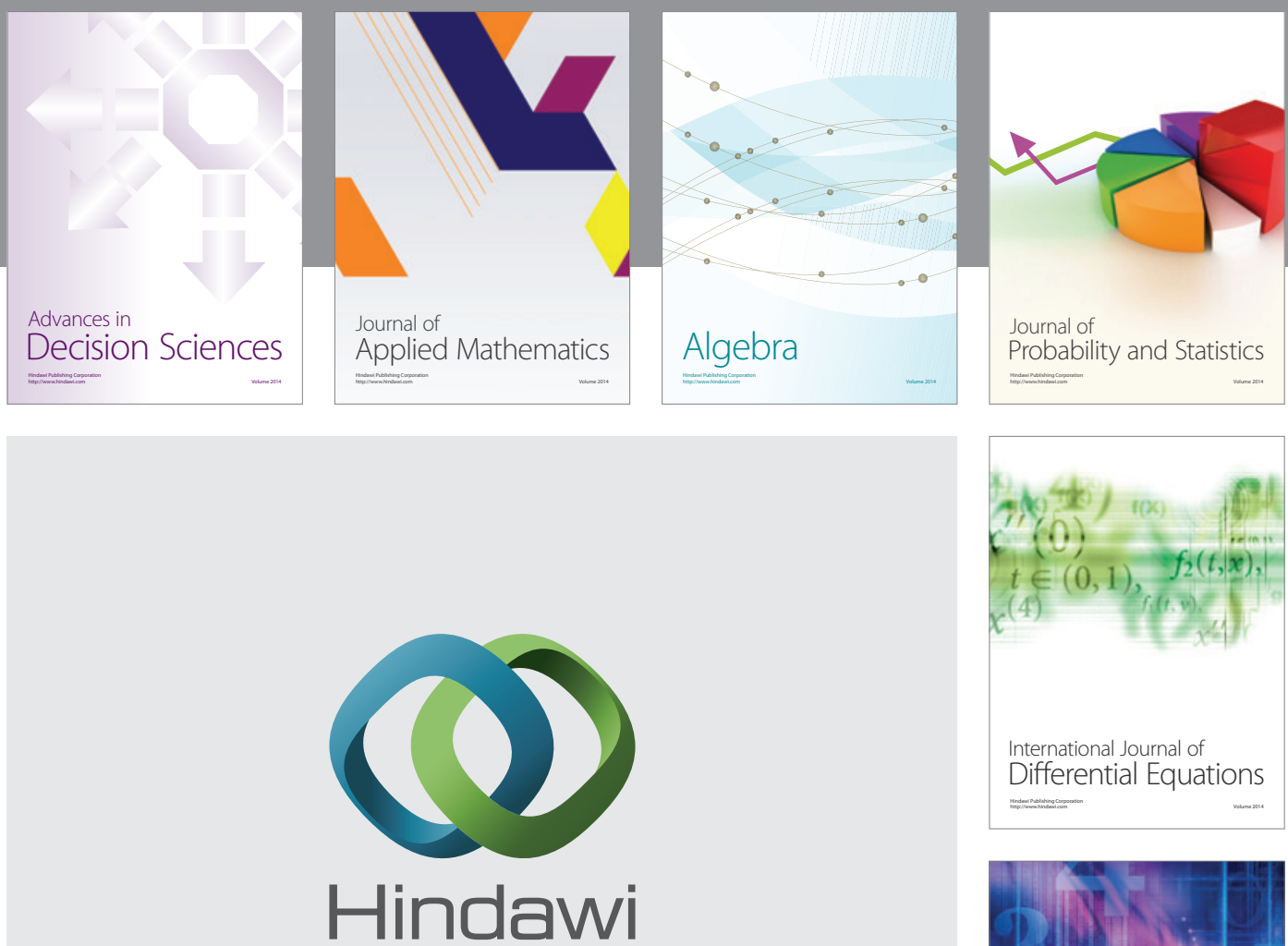

Submit your manuscripts at http://www.hindawi.com
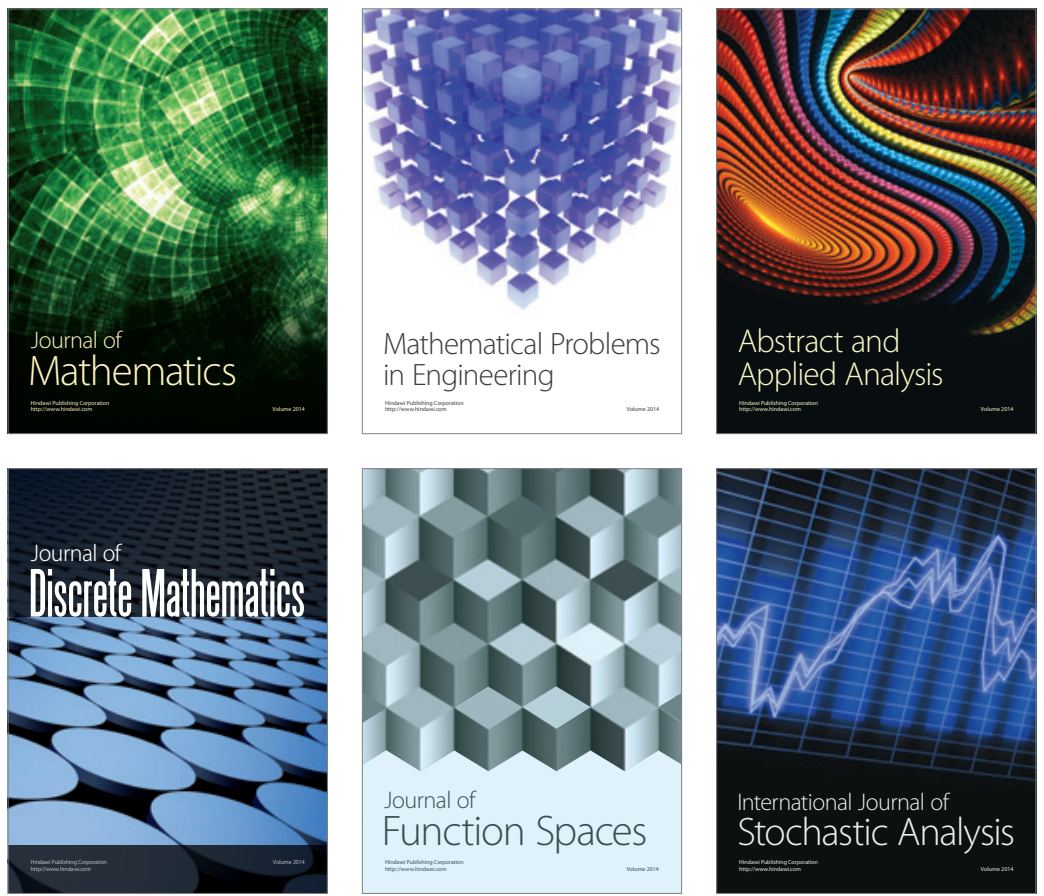

Journal of

Function Spaces

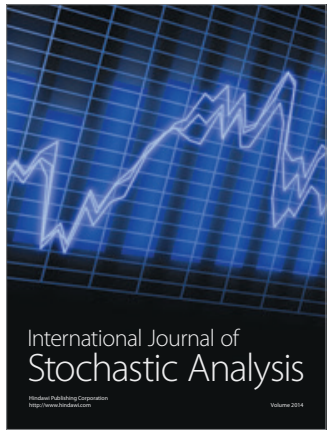

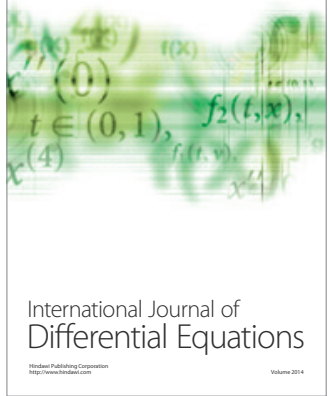
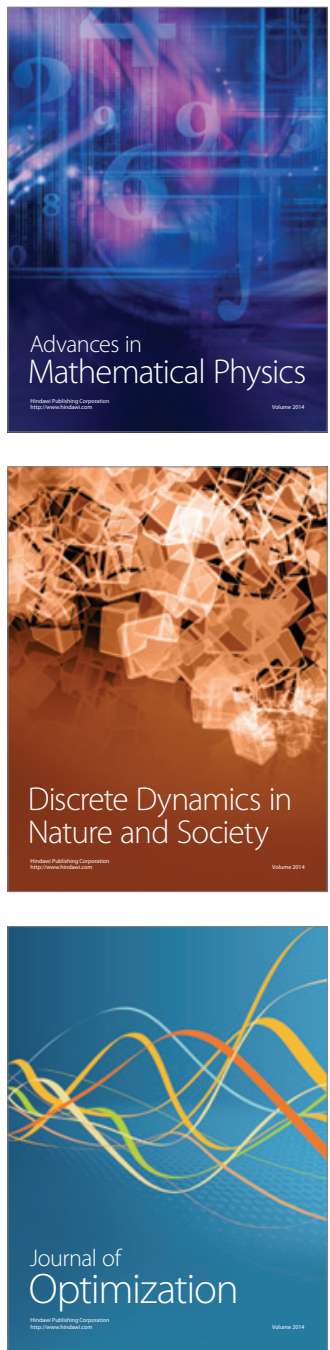